225 AN ORAL, SELECTIVE INHIBITOR OF TYROSINE KINASE 2, BMS-986165, IMPROVES MOLECULAR, CELLULAR, AND CLINICAL BIOMARKERS ASSOCIATED WITH EFFICACY IN MODERATE TO SEVERE PSORIASIS

${ }^{1}$ Ian Catlett ${ }^{*},{ }^{1}$ Sarah Hu, ${ }^{1}$ Subhashis Banerjee, ${ }^{2}$ Kenneth Gordon, ${ }^{3}$ James Krueger. ${ }^{1}$ BristolMyers Squibb; ${ }^{2}$ Medical College of Wisconsin; ${ }^{3}$ Rockefeller University Hospital, The Rockefeller University

\subsection{6/lupus-2019-Ism.225}

Background Psoriasis, a chronic immune-mediated inflammatory disease dependent upon the interleukin (IL)-23/TH17 pathway, is thought to be initiated through plasmacytoid dendritic cell activation and induction of type I interferons. BMS-986165 is a novel tyrosine kinase 2 (TYK2) inhibitor that blocks signal transduction of IL-23, IL-12, and type I interferons. BMS-986165 selectivity for TYK2, compared with Janus kinases (JAKs) 13, is driven by binding to its pseudokinase domain, rather than the conserved kinase domain.

Methods BMS-986165 was evaluated in a randomized, placebo-controlled, dose-ranging trial in 267 patients with moderate to severe psoriasis. Dose- and time-dependent effects on laboratory parameters indicative of non-selective inhibition of JAKs 13 were assessed. In an optional substudy, 37 patients provided biopsies, which were assessed from healthyappearing skin on Day 1 and from lesional skin on Days 1, 15 , and 85 for changes in the IL-23, IL-12, and type I interferon pathways by QRTPCR, RNA sequencing, and immunohistochemistry.

Results All BMS-986165 treatment groups, except $3 \mathrm{mg}$ every other day (QOD), achieved superiority versus placebo in the proportion of patients achieving Psoriasis Area and Severity Index 75 after 12 weeks of treatment (primary endpoint): $3 \mathrm{mg}$ QOD, 9.1\%; $3 \mathrm{mg}$ daily (QD), 38.6\%; $3 \mathrm{mg}$ twice daily (BID), 68.9\%; $6 \mathrm{mg} \mathrm{BID}, 66.7 \%$; and $12 \mathrm{mg} \mathrm{QD}$, $75.0 \%$ vs $6.7 \%$ with placebo. Mean levels of factors impacted with JAK 13 inhibition, including hemoglobin, total cholesterol, neutrophils, platelets, total lymphocytes, natural killer, and B cells, were not affected by BMS-986165. Markers of the IL-23 pathway including IL-17(A/F), S100A8/ 9, IL-22, and -defensin returned to non-lesion levels dosedependently. Interferon and IL-12 pathway genes were normalized; keratinocyte dysregulation markers keratin-16 and 10 , and late cornified envelope genes, returned toward nonlesion levels with effective doses.

Conclusions Clinical efficacy with BMS-986165 was associated with decreases in IL-23/TH17 and interferon pathway markers. TYK2 selectivity was confirmed by lack of effect on clinical biomarkers of JAK 13 inhibition. BMS-986165 has promising efficacy in psoriasis, and a distinct selectivity profile that warrants further investigation.

Funding Source(s): Bristol-Myers Squibb

\section{COMORBIDITY IN SYSTEMIC LUPUS ERYTHEMATOSUS}

${ }^{1}$ Bugra Egeli*, ${ }^{1}$ Asli Ece Soykut, ${ }^{2}$ Serdal Ugurlu. ${ }^{1}$ stanbul University-Cerrahpasa, Cerrahpasa Medical Faculty; ${ }^{2}$ Division of Rheumatology, Department of Internal Medicine, Cerrahpasa Medical Faculty, Istanbul University-Cerrahpasa

10.1136/lupus-2019-Ism.226
Abstract 226 Table 1 Prognostic factors of SLE patients in 2009 and 2018

\begin{tabular}{|c|c|c|c|}
\hline & $2009, n(\%)$ & $2018, n(\%)$ & $p$ value \\
\hline Age & $41 \pm 7$ & $53 \pm 8.76$ & \\
\hline AMI/Stroke & $\mathrm{n} / \mathrm{a}$ & 10 (15.38) & $\mathrm{n} / \mathrm{a}$ \\
\hline Cancer & $\mathrm{n} / \mathrm{a}$ & $2(3.08)$ & $\mathrm{n} / \mathrm{a}$ \\
\hline Diabetes & $2(2.13)$ & 8 (12.31) & 0.009 \\
\hline Hypertension & $33(35.11)$ & 37 (56.92) & 0.006 \\
\hline Angina & $\mathrm{n} / \mathrm{a}$ & $16(26.23)$ & $\mathrm{n} / \mathrm{a}$ \\
\hline Total & 94 & 65 & \\
\hline
\end{tabular}

Background In Systemic Lupus Erythematosus (SLE), cardiovascular mortality is important. In our previous study, we observed atherosclerotic plaque formation in carotid and/or femoral artery in SLE patients. ${ }^{1}$ The aim of this study was to assess the comorbidity of the patients diagnosed with SLE over 10 years.

Methods The sample group is a subset of 2009 study. In 2009 , the patients who already had myocardial infarction or cancer diagnosis were excluded. The patients were interviewed with polar questions of whether they were diagnosed with acute myocardial infarction (AMI), cerebrovascular events, cancer, diabetes, and hypertension.

Results We studied 65 patients $(56 \mathrm{~F}, 9 \mathrm{M}$; mean age: 53 $\pm 8.76)$ with SLE diagnosis.

In 2009, out of 94 SLE patients we included in the study, $2(2.13 \%)$ of the patients were diagnosed with diabetes, and $33(35.11 \%)$ with hypertension. However, in 2018, out of the 65 patients we reached, $8(12.31 \%)$ with diabetes, and 37 $(56.92 \%)$ with hypertension. The increase in diabetes and hypertension was found significant. $(p=0.009$ and 0.006$)$ The table 1 shows the percentages of newly occurred cancer, angina pectoris, and AMI/Stroke events.

Conclusions In 10 year follow-up, the significant increase in hypertension and diabetes can show us a sign of atherosclerotic plaque formation. However, the increased hypertension and diabetes ratio can still be due to increased age.

Funding Source(s): None

\section{REFERENCE}

1. Ugurlu S, Seyahi E, Cetinkaya F, Ozbakir F, Balci H, Ozdogan H. Intima-media thickening in patients with familial Mediterranean fever. Rheumatology (Oxford). 2009 August;48(8):911-5.

\section{THE LUPUS SEVERITY INDEX ACCURATELY IDENTIFIES PATIENTS WITH SEVERE SLE IN A MULTI-ETHNIC COHORT}

${ }^{1}$ Christine Peschken* ${ }^{2}{ }^{2}$ Carol Hitchon, ${ }^{2}$ David Robinson, ${ }^{2}$ Annaliese Tisseverasinghe, ${ }^{2} \mathrm{Hani}$ El-Gabalawy. ${ }^{1}$ Faculty of Medicine, Department of Internal Medicine, University of Manitoba; ${ }^{2}$ University of Manitoba

\subsection{6/lupus-2019-Ism.227}

Background The Lupus Severity Index (LSI) ${ }^{1}$ proposes to stratify patients by disease severity for clinical research. The LSI ranges from $0-10$, is calculated using ACR classification criteria (ACRc) and demonstrated high predictive accuracy for severity anchored to major immunosuppressive drug use. 

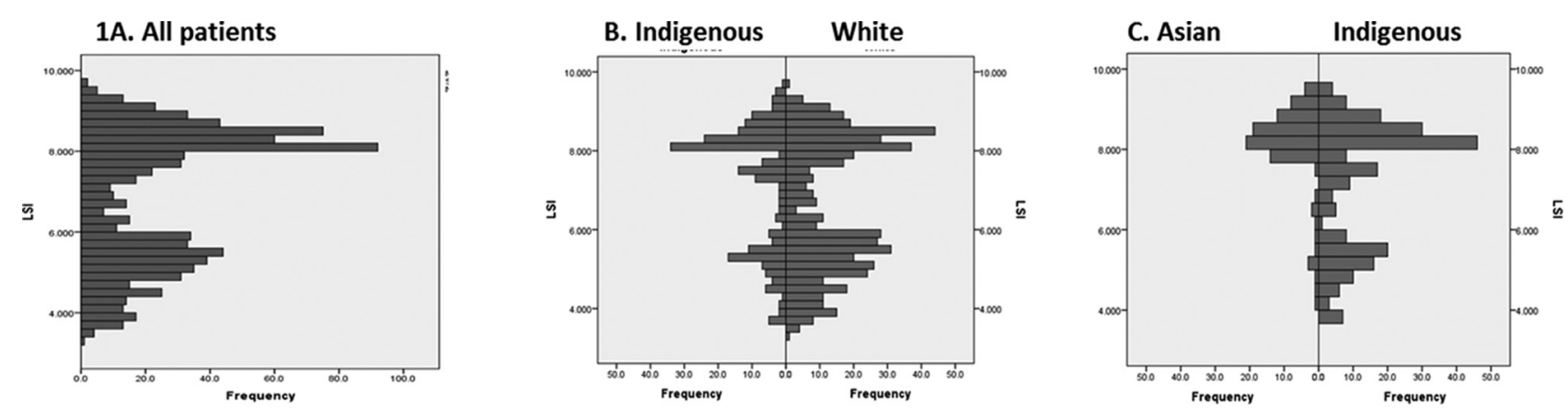

Abstract 227 Figure 1 Distribution of Lupus Severity Index in all Patients and in different ethnic groups

We investigated the performance and characteristics of the LSI in a large multiethnic lupus cohort.

Methods Patients from a single academic center were followed from 1990-2016 using a custom database. Records of all SLE patients were abstracted. Variables included birthdate, diagnosis date, ethnicity, ACRc, SLICC Damage Index (SDI), treatment and date of death. Ethnicity was categorized into White (WHI), Asian (ASN), Indigenous (IND), and Other. The LSI was calculated from ACRc, and compared between ethnicities and demographic variables known to be associated with severe SLE using t-tests, ANOVA, Pearson correlation coefficient and logistic regression.

Results 832 SLE patients were identified: 497 (60\%) WHI; 220 (26\%) IND; 91 (11\%) ASN; 24 (3\%) Other. Mean age was 49 years, mean disease duration 15 years, 90\% female, mean age at diagnosis $35 ; 163(20 \%)$ of patients had died. The mean LSI was 6.9, range 3.2-9.7. The distribution of the LSI was similar to that in the original dataset (figure 1A) and the area under the ROC curve, measured against prescription of major immunosuppressive drugs, was $0.69(95 \%$ CI $0.65-0.73)$. LSI was higher in males compared to females (7.3 vs. 6.9; p-0.019), and was negatively associated with onset age (Onset $<18$ years $\mathrm{LSI}=7.8 ; 18-50$ years $\mathrm{LSI}=6.8$; $>50$ years $\mathrm{LSI}=6.6 ; \mathrm{p}<0.001)$. LSI correlated with SDI(Pearson $0.28, \mathrm{p}<0.001)$, and was a predictor of accruing any damage (SDI>1) (OR1.2 95\% CI 1.1-1.3).LSI was higher in non-whites compared to whites: WHI LSI=6.6; IND LSI=7.2; Other LSI=7.3; ASN LSI 8.1; $\mathrm{p}<0.001)$. LSI was a predictor of early mortality (Death at age $<50$, or disease duration <10 years): OR 1.2; 95\% CI 1.0-1.3). The distribution of the LSI varied by ethnic group with more uniformly severe disease in ASN patients (figure 1B, C) compared to WHI and IND.

Conclusions Similar to the original publication, higher LSI correlated with male sex, younger onset age, and non-white ethnicity; all groups shown to have more severe SLE. LSI was also a predictor of damage and early mortality. In addition we also found the distribution of LSI to differ between ethnicities. These findings confirm the utility of the LSI in stratifying patients by severity, and supports further exploration of the LSI to investigate contributors to severe SLE.

\section{REFERENCE}

1. Bello GA et al. Lupus Science \& Medicine 2016.

Funding Source(s): None

\section{VERDINEXOR, AN INHIBITOR OF THE NUCLEAR EXPORT PROTEIN EXPORTIN-1 PREVENTS LUPUS PROGRESSION BY LIMITING GERMINAL CENTER FORMATION AND DEVELOPMENT OF AUTOREACTIVE ANTIBODY SECRETING CELLS}

${ }^{1}$ Javier Rangel-Moreno, ${ }^{2}$ Nida Meednu, ${ }^{3}$ Douglas G Widman*, ${ }^{3}$ Savanna Gornisiewicz, ${ }^{3}$ Sharon Tamir, ${ }^{4}$ Jennifer Anolik. ${ }^{1}$ Division of Allergy, Immunology and Rheumatology, Department of Medicine, URMC; ${ }^{2}$ University of Rochester; ${ }^{3}$ Karyopharm Therapeutics; ${ }^{4}$ Allergy-Immunology-Rheumatology; University of Rochester Medical Center

\subsection{6/lupus-2019-Ism.228}

Background Systemic lupus erythematosus (SLE) is a complex autoimmune disease characterized by simultaneous activation of the innate and adaptive arms of the immune system. Recently, the nuclear export protein exportin-1 (XPO1) has surfaced as an attractive target for the treatment of SLE. Selective Inhibitor of Nuclear Export (SINE) compounds such as verdinexor (KPT-335) are potent, orally available XPO1 inhibitors. SINE compounds inhibit the nuclear export of over 220 cargoes, and this pleiotropic effect on multiple signaling pathways exerts apoptotic and anti-inflammatory effects, particularly in dampening NF-B transcriptional activity. Based on previous studies with SINE compounds, we sought to define the minimal amount and frequency of verdinexor treatment necessary to maintain disease inhibition.

Methods To evaluate the minimal efficacious dose of verdinexor in SLE, cohorts of mice with established disease were dosed orally twice weekly with $7.5 \mathrm{mg} / \mathrm{kg}$ verdinexor or vehicle for eight weeks. Thereafter, various administration schedules of verdinexor $(7.5 \mathrm{mg} / \mathrm{kg})$ plus or minus bortezomib $(0.75 \mathrm{mg} / \mathrm{kg})$ a proteasome inhibitor (PI) known to eliminate autoreactive antibody-secreting cells (ASC) were administered for 4 weeks to examine their ability to control recurrent disease. We used flow cytometry and ELISPOT to enumerate ASC in the spleen and bone marrow (BM) and immunofluorescence to visualize germinal centers (GC) in spleen.

Results Therapy with verdinexor twice weekly significantly inhibited disease progression (GC: Control $=622499.06$ 338011.04 vs KPT-335 $=100952.4462652 .09, \mathrm{p}=0.0033$. plasmablasts: $\quad$ Control $=263790.82 \quad 148146.57$ vs $\quad$ KPT$335=20967.31$ 19009.25, $\mathrm{p}=0.0027$. plasma cells: Control $=62078.36 \quad 22539.49$ vs $\quad$ KPT-335 $=21277.82 \quad 13909.45$, $\mathrm{p}=0.0056$ ). Thus, we observed significantly decreased levels of GC B cells, plasma cells and plasmablasts in the BM and the 